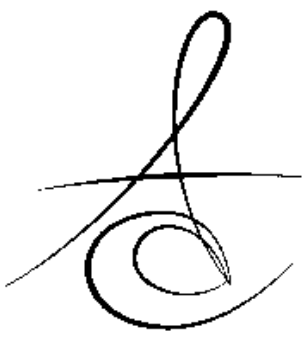

\title{
WAVEONE, RECIPROC VE TWISTED FILE ADAPTIVE DÖNER SİSTEM EĞELERİN ÇİFT KURVATÜRLÜ (S-ŞEKİLLİ) KANALLARDA DÖNGÜSEL YORULMA DİRENÇLERİNİN KARŞILAŞTIRILMASI
}

\section{COMPARISON OF THE CYCLIC FATIGUE RESISTANCE OF WAVEONE, RECIPROC AND TWISTED FILE ADAPTIVE FILES IN CANALS WITH A DOUBLE CURVATURE (S-SHAPED)}

\author{
Dr. Öğr. Üyesi Mehmet ADIGÜZEL ${ }^{*}$
}

\section{Dr. Öğr. Üyesi Pelin TÜFENKÇi்}

Makale Kodu/Article code: 3265

Makale Gönderilme tarihi: 04.01.2017

Kabul Tarihi; 12.06.2017

\section{öz}

Amaç: Bu çalışmanın amacl, WaveOne (Dentsply Maillefer, Ballaigues, İsviçre), Reciproc (VDW, Münih, Almanya) ve Twisted File Adaptive (Axis/SybronEndo, Orange, CA, ABD) döner sistem nikel titanyum ( $\mathrm{Ni}-\mathrm{Ti}$ ) eğelerin çift kurvatürlü (S-şekilli) kanallarda döngüsel yorgunluğa bağlı kırılma dirençlerinin karşılaştırılmasıdır.

Gereç ve Yöntem: Bu çalışmada 45 adet (WaveOne primary, Reciproc R25 ve Twisted File Adaptive M-L1) eğe kullanıldı. Her bir sistemden 15 eğe kırılıncaya kadar çift kurvatürlü (S-şekilli) yapay bir kanalda döndürüldü. Kırılıncaya kadar olan tur sayısı hesaplandı ve kırılmış parçaların uzunluğu kaydedildi. Veriler, ANOVA ve Tukey post hoc testleri kullanılarak istatistiksel olarak analiz edildi. İstatistiksel anlamlılık düzeyi $p<0.05$ olarak belirlendi.

Bulgular: Reciproc R25 eğeleri hem apikal hem de koranal kurvatürde, WaveOne primary ve Twisted File Adaptive M-L1 eğelerine göre döngüsel yorgunluğa karşı daha dirençli bulundu $(p<0.05)$. WaveOne primary ve Twisted File Adaptive M-L1 eğeleri arasında her iki kurvatürde de istatistiksel olarak anlamlı farklılık saptanmadı $(p>0.05)$. Bütün gruplar arasında aletlerin kırılmış fragmanlarının uzunluğu istatistiksel olarak benzer bulundu ( $p>0.05)$.

Sonuç: Reciproc R25 eğeler, WaveOne primary ve Twisted File Adaptive eğelere göre çift kurvatürlü kanallarda kırılmaya karşı daha dirençli bulundu.

Anahtar Kelimeler: Döngüsel yorgunluk, Reciproc, WaveOne, S-şekilli kanal

\section{ABSTRACT}

Aim: The purpose of this study was to compare the cyclic fatigue resistance of WaveOne (Dentsply Maillefer, Ballaigues, Switzerland), Reciproc (VDW, Münich, Germany) and Twisted File Adaptive (Axis/SybronEndo, Orange, CA, USA) nickel titanium (Ni-Ti) systems in canals with a double curvature.

Materials and Methods: Forty five rotary NiTi files (WaveOne primary, Reciproc R25 and Twisted File Adaptive M-L1) were used in this study. Fifteen files from each system were rotated in canals with a double curvature until fracture. The number of cycles to fracture was calculated and the length of each fractured fragment was recorded. The data were statistically analysed using ANOVA and Tukey post hoc tests. The statistical significance level was set at $p<0.05$.

Results: The Reciproc R25 file had higher cyclic fatigue resistance than WaveOne primary and Twisted File Adaptive in the apical and coronal curvatures $(p<0.05)$. There was no significant distinction in the cyclic fatigue resistance of the WaveOne primary and Twisted File Adaptive files in either the apical or coronal curvature. The length of the fractured part of the instruments was similar among all the groups $(p>0.05)$.

Conclusion: The Reciproc R25 file indicated better cyclic fatigue resistance than WaveOne primary and Twisted File Adaptive in an artificial canal with an S-shape.

Keywords: Cyclic fatigue, Reciproc, WaveOne, S-shaped canal.

* Mustafa Kemal Üniversitesi, Diş Hekimliği Fakültesi Endodonti A.D Hatay 


\section{GİRİş}

Yüksek esnekliği ve kesme verimliliği nedeniyle nikel-titanyum (Ni-Ti) döner aletler kök kanallarını şekillendirmek için yaygın olarak kullanılmaktadır. ${ }^{1-4}$ Döner aletlerin klinik kullanımdaki pratikliği tercih sebebi olsa da, iki farklı mekanizmaya (torsiyonel ve döngüsel yorgunluk) bağlı olarak Ni-Ti eğelerde oluşan alet kırılması tedaviyi olumsuz etkilemektedir. ${ }^{5-7}$ Torsiyonel yorgunluk, eğenin ucu kanal içerisinde sıkıştığı zaman sap kısmının dönme hareketinin devam etmesi sonucunda meydana gelmektedir. Döngüsel yorgunluk ise eğenin belirli bir bölgesinin tekrarlayan sıkışma ve gerilme kuvvetlerine maruz kalmasıyla oluşmaktadır. ${ }^{8-10}$ Döngüsel yorgunluk klinik kullanım sırasında NiTi döner aletlerin kırılmasının ana sebeplerinden biridir. ${ }^{11}$

Döngüsel yorgunluk testleri statik ve dinamik olmak üzere iki farklı yöntemle yapılmaktadır. Statik döngüsel yorgunluk modellerinde çalışma uzunluğu sabit tutulmakta ve eğe yapay kanal içerisinde kırılıncaya kadar döndürülmektedir. Eğelerin ömrünü uzattığı bildirilen dinamik modelde ise eğe dönerken aynı zamanda aksiyal yönde ileri geri hareket etmektedir. ${ }^{12}$

WaveOne (Dentsply Maillefer, Ballaigues, İsviçre) ve Reciproc (VDW, Münih, Almanya) eğelerin 2011 yılında piyasaya çıkmasının ardından tek eğe sistemleri popülerlik kazanmıştır. Her iki sistemin eğeleri ' $M$ ' wire teknolojisinden üretilmektedir. ${ }^{13}$ İki sistemde kullanılan resiprokasyon hareketi, rotasyon hareketine göre aletlerin kanal içinde vidalanma riskini, maruz kaldıkları sıkışma ve gerilme kuvvetlerini azaltmaktadır. Ancak her iki sistem farklı dönme açı- sına ve hızlarına sahiptir. (WAVEONE ALL modu için $350 \mathrm{rpm}, 170^{\circ}$ saat yönünün tersine, $50^{\circ}$ saat yönün- de; RECİPROC ALL modu için $300 \mathrm{rpm}, 150^{\circ}$ saat yönünün tersine, $30^{\circ}$ saat yönünde) Twisted File Adaptive (Axis/ SybronEndo, Orange, $A B D$ ) eğeleri ise resiprokasyon ve rotasyon hareketini kombine eden bir sistemdir. ${ }^{14}$ Adapte olabilen hareket teknolojisi ile düşük bir basınç olduğunda kanal eğesi rotasyon hareketi yapmaktadır. Diğer yandan, eğenin kanal içerisinde sıkıştığı durumlarda veya yüksek bir basınç olduğunda eğe resiprokasyon hareketine geçmektedir. ${ }^{15}$

Güncel olarak, eğelerin çift kurvatürlü kanallardaki kırıma dirençleri ile ilgili olarak literatürde sınırlı sayıda çalışma bulunmaktadır. Bu çalışmanın amacı,
WaveOne, Reciproc ve Twisted File Adaptive döner sistem Ni-Ti eğelerin çift kurvatürlü (S-şekilli) kanallarda döngüsel yorgunluğa bağlı kırılma dirençlerinin karşılaştırımasıdır.

\section{GEREÇ VE YÖNTEM}

Bu çalışmada 15 adet WaveOne primary (boyut 25, .08 taper), 15 adet Reciproc R25 (boyut 25, .08 taper) ve 15 adet Twisted File Adaptive M-L1 (boyut 25, .08 taper) Ni-Ti eğe kullanıldı. Deneyden önce her bir eğe stereo mikroskop altında kusur ve şekil bozuklukları açısından kontrol edildi. Tüm kusurlu eğeler yenileri ile değiştirildi.

Her bir sistemin eğesi, S-şekilli yapay kanal (çap, $1.4 \mathrm{~mm}$ ve uzunluk, $18 \mathrm{~mm}$ ) içeren paslanmaz çelik bir blok kullanılarak döngüsel yorulma testine tabi tutuldu. Kullanılan blok daha önce bu alanda yapılan çalışmalara göre hazırlandı. ${ }^{16,17}$ Bloğun koronal kurvatürü $60^{\circ}$ eğrilik açısına ve $5 \mathrm{~mm}$ yarıçapa sahiptir ve aletin ucundan $8 \mathrm{~mm}$ uzaklıktadır; apikal kurvatürü $70^{\circ}$ eğrilik açısına, $2 \mathrm{~mm}$ yarıçapa ve aletin apikal ucundan $2 \mathrm{~mm}$ uzaktaki bir merkeze sahiptir. (Şekil 1)

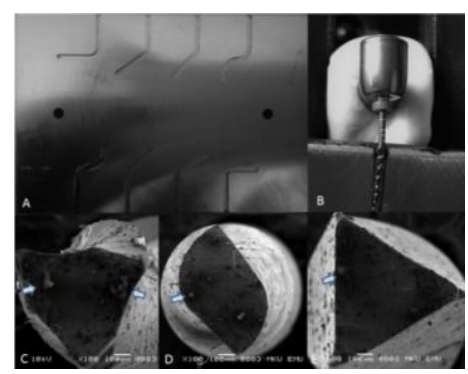

Şekil 1.

(A) Metal kanal bloğun fotoğrafik görüntüsü

(B) Döngüsel yorgunluk test cihazının fotoğrafik görüntüsü

(C) Kırık WaveOne eğelerin örnek SEM görüntüsü

(D) Kırık Reciproc eğelerin örnek SEM görüntüsü

(E) Kırık Twisted File Adaptive eğelerin örnek SEM görüntüsü

WaveOne ve Reciproc eğelerin döngüsel yorgunlukları tork-kontrollü bir motorda (VDW Silver; VDW, Münih, Almanya), Twisted File Adaptive eğeleri ise kendi motorlarında (Elements Motor; Sybron Endo, Orange, CA, ABD) test edildi. Üreticilerin önerileri doğrultusunda WaveOne eğelerde WaveOne ALL modu, Reciproc eğelerde Reciproc ALL modu ve Twisted File Adaptive eğelerde sistemin kendi özel modu kullanıldı. Eğelerin sürtünmesini azaltmak için sentetik bir yağ (WD-40 Company, Milton Keynes, İngiltere) kulanıla- 
rak kayganlık sağlandı. Çalışmada klinik kullanımı simüle etmek için devamlı ileri-geri hareketler kullanıld. ${ }^{18}$ Her bir eğenin kırılma süresi hesaplandı. Kırılıncaya kadar olan tur sayısı; dönme hızı (rpm) $\times$ kırılma süresi (saniye) / 60 formülü ile hesaplandı. Ayrıca, eğer alet kırıması ilk olarak apikal kurvatürde ortaya çıktı ise, kırıma zamanı ve tur sayısı koronal parça içinde kaydedildi. Kırıımış parçanın uzunluğu milimetre cinsinden ölçüldü.

Test edilen aletlerin kırıma yüzeylerinin örnek SEM görüntüleri Şekil 1'de gösterildi. Veriler, ANOVA ve Tukey post hoc testleri kullanılarak istatistiksel olarak analiz edildi. İstatistiksel anlamlılık düzeyi $p<$ 0.05 olarak belirlendi.

\section{BULGULAR}

Her bir grubun gerçekleştirdiği tur sayısı ve test sonunda kırılan eğe uzunlukları Tablo 1 'de verildi.
İstatisiksel olarak Reciproc R25 eğeleri hem

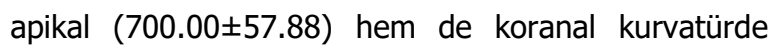
$(807.66 \pm 139.76)$, WaveOne primary (apikal 449.29 \pm 46.22 ; koronal $530.67 \pm 94.91$ ) ve Twisted File Adaptive M-L1 (apikal 410.00 \pm 40.00 ; koronal $477.33 \pm 83.53)$ eğelerine göre döngüsel yorgunluğa karşı daha dirençli bulundu $(p<0.05)$. WaveOne primary and Twisted File Adaptive M-L1 eğeleri arasında istatistiksel olarak an- lamlı farklılık saptanmadı $(p>0.05)$. Bütün gruplar arasında aletlerin kırılmış bölümünün uzunluğu istatistiksel olarak benzer bulun$\mathrm{du}(p>0.05)$. SEM incelemesinde gözlenen yorulma çizgilerinin varlı̆ı ve dairesel aşınmanın olmaması kırımaların döngüsel yorgunluğa bağlı olarak gerçekleştiğini göstermektedir. (Şekil 1)

Tablo 1. Döngüsel yorgunluk testi sırasında eğelerin başarısız oluncaya kadar yaptığı tur sayısı (ortama ve standart sapma) ve test sonucunda kırılan eğelerin uzunluğu $(\mathrm{mm})$

\begin{tabular}{|c|c|ccc|cccc|ccc|cc|}
\hline \multirow{2}{*}{ Grup } & \multirow{2}{*}{$\mathbf{n}$} & \multicolumn{4}{|c|}{ Koronal Kurvatür } & \multicolumn{4}{c|}{ Apikal Kurvatür } \\
& & \multicolumn{2}{|c|}{ Tur Sayıs } & \multicolumn{3}{c|}{ Kırık Alet Uzunluğu } & \multicolumn{2}{c|}{ Tur Sayısı } & \multicolumn{1}{c|}{ Kırık Alet Uzunluğu } \\
\hline WaveOne & 15 & 530.67 & \pm & 94.91 & 6.50 & \pm & 0.42 & 449.29 & \pm & 46.22 & 2.18 & \pm & 0.27 \\
Reciproc & 15 & 807.66 & \pm & 139.76 & 6.37 & \pm & 0.56 & 700.00 & \pm & 57.88 & 2.20 & \pm & 0.22 \\
Twisted File Adaptive & 15 & 477.33 & \pm & 83.53 & 6.24 & \pm & 0.52 & 410.00 & \pm & 40.00 & 2.34 & \pm & 0.24 \\
\hline
\end{tabular}

\section{TARTIŞMA}

Endodontik aletlerin kesiti, kompozisyonu ve dizaynı gibi birçok faktör aletlerin performansı ve kırılma direnci üzerinde önemli etkilere sahiptir. ${ }^{14}$ Klinisyenlerin endodontik alet özelliklerinin farkında olması tedavi başarı oranını artıracaktır. Kırılmaya karşı daha dirençli bir alet seçmek, klinik koşullarda meydana gelen eğe kırıklarını azaltacaktır. ${ }^{19}$

Diş kurvatür derecesi eğenin dönme yorgunluğuna bağlı olarak kırılmasını etkileyen ana değişkenlerden biridir. Klinik koşullar altında aynı kök kanalında iki kurvatür (S şekilli) mevcut olabilir. S-şekilli kanallar, $\mathrm{Ni}$-Ti aletleri kullanmanın en zorlu klinik koşullarından biridir ve geleneksel radyografide izlenmesi oldukça zordur. ${ }^{20}$ Araştırıcılar Ni-Ti aletlerin S-şekilli kanallarda kullanıldığında yorulmanın çok hızlı bir şekilde gerçekleştiğini belirtmiştir. ${ }^{17,20}$
Literatürde endodontik aletlerin döngüsel yorulma direnci, çeşitli çalışma dizaynları kullanılarak incelenmiştir. Klinik koşulları taklit eden dönme yorgunluk testleri kullanmak araştırmacılar için ideal bir hedef olmuştur. Bununla birlikte, doğal dişlerdeki kök kanal sisteminin standartlaştırılması ve her bir alet için test koşullarının tekrarlanabilirliğinin sağlanması bu test yönteminde mümkün olmamıştır. ${ }^{16}$ Ancak bu test analizi için paslanmaz çelik bir blokta yapay olarak simüle edilmiş kanallar statik ve dinamik modelde güvenilir bir şekilde kullanılmıştır. ${ }^{21}$

Bu çalışmada farklı üç markanın aynı boyut ve taperdaki (apikal 3mm) eğelerinin çift kurvatürlü kanallarda döngüsel yorgunluk dirençleri karşılaştırıldı. Çalışma için piyasada resiprokosyan hareketi yapan en önemli iki eğe (Reciproc ve WaveOne) ile adapte olabilen hareket teknolojisine sahip Twisted File Adaptive eğeler seçildi. Çalışmanın sonuçlarına göre Reciproc

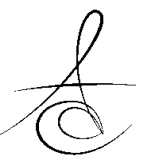


R25 eğesi, WaveOne primary ve Twisted File Adaptive $M-L 1$ eğelerine göre döngüsel yorgunluğa karşı daha dirençli bulunmuştur.

Kullanılan eğeler aynı boyut ve aynı apikal taperda olmasına rağmen farklı kesitlere ve kinematiğe sahiptir. Reciproc R25 eğesi çift taraflı keskin bir Sşekilli geometriye, WaveOne primary eğesi konveks üçgen enine-kesitli geometriye, Twisted File Adaptive eğesi ise eşkenar üçgen enine-kesitli bir geometriye sahiptir. ${ }^{14}$ Bununla birlikte Reciproc (300 rpm), WaveOne (350 rpm) ve Twisted File Adaptive (400 rpm) eğelerinin farklı hızla çalıştığı birçok çalışmada belirtilmiştir. ${ }^{16,22}$ Aletlerin farklı kesitsel tasarımları ile farklı kinematikleri çalışmanın sonuçlarını etkilemiş olabilir. ${ }^{23}$ Daha önceki çalışmalarda, Reciproc R25'in WaveOne Primary'e göre döngüsel yorgunluğa karşı daha dirençli olduğunu gösterilmiştir. ${ }^{22,24} \mathrm{Bu}$ sonuç, bizim çalışmamızın sonuçları ile uyumludur. Ertaş ve ark. ${ }^{23}$ yapmış oldukları çalışmada WaveOne Primary ve Twisted File Adaptive eğeleri arasında kırılma dirençleri arasında anlamlı bir farklılık olmadığını belirtmişlerdir. Mevcut çalışmamızda da buna benzer sonuçlar elde edilmiştir.

$\mathrm{Bu}$ makalenin verilerine göre döngüsel yorgunluk direnci ile üreticiler tarafından belirtilen hız arasında bir ilişki bulunamamıştır. En yüksek kırıma direnci en düşük hıza sahip Reciproc eğelerde (300 rpm) gözlenirken, daha yüksek hızlara sahip olan WaveOne (350 rpm) ve Twisted File Adaptive (400 rpm) eğelerde ise daha düşük kırılma direnci görülmüştür. Bu sonuçlar Higuera ve ark. ${ }^{22}$ yapmış oldukları çalışmanın sonuçları ile desteklenmektedir.

\section{SONUÇ}

Çalışmamızın limitleri dahilinde Reciproc R25 eğeler, WaveOne primary and Twisted File Adaptive eğelere göre çift kurvatürlü kanallarda kırılmaya karşı daha dirençli bulunmuştur.

Mehmet Adıgüzel, ORCID ID: 0000-0002-9363-6264

Pelin Tüfenkçi, ORCID ID: 0000-0001-9881-5395

\section{KAYNAKLAR}

1. Arias A, Perez-Higueras JJ, de la Macorra JC. Differences in cyclic fatigue resistance at apical and coronal levels of Reciproc and WaveOne new files. J Endod. 2012;38:1244-8.

2. Perez-Higueras JJ, Arias A, de la Macorra JC. Cyclic fatigue resistance of $\mathrm{K} 3, \mathrm{~K} 3 \mathrm{XF}$, and twisted file nickel-titanium files under continuous rotation or reciprocating motion. J Endod 2013;39:15858.

3. Capar ID, Ertas H, Arslan H. Comparison of cyclic fatigue resistance of nickel-titanium coronal flaring instruments. J Endod 2014;40:1182-5.

4. Göktürk $H$, Yücel $A C ̧$, Şişman $A$. The shaping ability of five different nickel-titanium rotary instruments in simulated root canals. J Dent Fac Atatürk Uni 2014;24:58-66

5. Knowles KI, Hammond NB, Biggs SG, Ibarrola JL. Incidence of instrument separation using LightSpeed rotary instruments. J Endod 2006;32:14-6.

6. Parashos $\mathrm{P}$, Messer HH. Rotary NiTi instrument fracture and its consequences. J Endod 2006;32:1031-43.

7. Sattapan B, Nervo GJ, Palamara JE, Messer HH. Defects in rotary nickel-titanium files after clinical use. J Endod 2000;26:161-5.

8. Bouska J, Justman B, Williamson A, DeLong C, Qian F. Resistance to cyclic fatigue failure of a new endodontic rotary file. J Endod 2012;38:667-9.

9. Kim HC, Kwak SW, Cheung GS, Ko DH, Chung SM, Lee W. Cyclic fatigue and torsional resistance of two new nickel-titanium instruments used in reciprocation motion: Reciproc versus WaveOne. J Endod 2012;38:5414.

10. Setzer FC, Bohme CP. Influence of combined cyclic fatigue and torsional stress on the fracture point of nickel-titanium rotary instruments. J Endod 2013;39:133-7.

11. Cheung GS, Peng B, Bian Z, Shen Y, Darvell BW. Defects in ProTaper S1 instruments after clinical use: fractographic examination. Int Endod J 2005;38:802-9. 
12. Dederich DN, Zakariasen $\mathrm{KL}$. The effects of cyclical axial motion on rotary endodontic instrument fatigue. Oral Surg Oral Med Oral Pathol 1986;61:192-6.

13. Ozyurek T. Cyclic Fatigue Resistance of Reciproc, WaveOne, and WaveOne Gold Nickel-Titanium Instruments. J Endod 2016; 42:1536-9.

14. Elsaka SE, Elnaghy AM, Badr AE. Torsional and bending resistance of WaveOne Gold, Reciproc and Twisted File Adaptive instruments. Int Endod ] 2017;50:1077-83.

15. Karatas E, Arslan H, Kirici DO, Alsancak M, Capar ID. Quantitative evaluation of apically extruded debris with Twisted File Adaptive instruments in straight root canals: reciprocation with different angles, adaptive motion and continuous rotation. Int Endod J 2016;49:382-5.

16. Topcuoglu HS, Duzgun S, Akti A, Topcuoglu G. Laboratory comparison of cyclic fatigue resistance of WaveOne Gold, Reciproc and WaveOne files in canals with a double curvature. Int Endod J 2017;50:713-7.

17. Al-Sudani D, Grande NM, Plotino G, et al. Cyclic fatigue of nickel-titanium rotary instruments in a double (S-shaped) simulated curvature. J Endod 2012;38:987-9.

18. De-Deus G, Leal Vieira VT, Nogueira da Silva EJ, Lopes $\mathrm{H}$, Elias CN, Moreira EJ. Bending resistance and dynamic and static cyclic fatigue life of Reciproc and WaveOne large instruments. J Endod 2014;40:575-9.

19. Gao Y, Gutmann JL, Wilkinson K, Maxwell R, Ammon D. Evaluation of the impact of raw materials on the fatigue and mechanical properties of ProFile Vortex rotary instruments. J Endod 2012;38:398-401.

20. Topcuoglu HS, Topcuoglu G, Akti A, Duzgun S. In Vitro Comparison of Cyclic fatigue resistance of ProTaper Next, HyFlex CM, OneShape, and ProTaper Universal Instruments in a canal with a double curvature. J Endod 2016;42:969-71.

21. Capar ID, Kaval ME, Ertas $H$, Sen BH. Comparison of the cyclic fatigue resistance of 5 different rotary pathfinding instruments made of conventional nickel-titanium wire, m-wire, and controlled memory wire. J Endod 2015;41:535-8.
22. Higuera O, Plotino G, Tocci L, Carrillo G, Gambarini G, Jaramillo DE. Cyclic fatigue resistance of 3 different nickel-titanium reciprocating instruments in artificial canals. J Endod 2015;41:913-5.

23. Ertaş H, Capar ID, Arslan H. Cyclic fatigue resistance of ProTaper Universal, Twisted File Adaptive, Reciproc and WaveOne systems. Turk Endod J 2016;1:30-4.

24. Plotino G, Grande NM, Testarelli L, Gambarini G. Cyclic fatigue of Reciproc and WaveOne reciprocating instruments. Int Endod J 2012;45:614-8.

\author{
Yazışma Adresi \\ Dr. Öğr. Üyesi Mehmet ADIGÜZEL \\ Mustafa Kemal Üniversitesi, \\ Diş Hekimliği Fakültesi, \\ Endodonti Anabilim Dalı, \\ Hatay, 31100, Türkiye \\ Telefon numarası: +90.326.229 1000-3017 \\ Fax numarası: +90.326.229 5654 \\ E-mail: dt.mehmetadiguzel@gmail.com
}

